\title{
Natriuretic Peptide Resistance of Mesenteric Arteries in Spontaneous Hypertensive Rat Is Alleviated by Exercise
}

\author{
Jun YU ${ }^{1^{*}}$, Bing ZHANG ${ }^{2^{*}}$, Xing-Li SU ${ }^{3^{*}}$, Ru TIE ${ }^{1^{*}}$, Pan CHANG ${ }^{*}$, Xue-Ce ZHANG ${ }^{1}$, \\ Jian-Bang WANG ${ }^{4}$, Ge ZHAO ${ }^{5}$, Miao-Zhang $\mathrm{ZHU}^{6}$, Hai-Feng ZHANG ${ }^{7}$, Bao-Ying CHEN \\ ${ }^{*}$ These authors contributed equally to this work. \\ ${ }^{1}$ Central Laboratory, Second Affiliated Hospital, Xi'an Medical University, Xi'an, China, \\ ${ }^{2}$ Department of Orthopedics, Second Affiliated Hospital, Xi' an Medical University, Xi' an, China, \\ ${ }^{3}$ Department of Pathophysiology, Xi' an Medical University, Xi' an, China, ${ }^{4}$ Department \\ of Cardiology, Second Affiliated Hospital, Xi'an Medical University, Xi'an, China, ${ }^{5}$ Department \\ of Anesthesiology, First Affiliated Hospital, School of Medicine, Xi'an Jiaotong University, Xi' an, \\ China, ${ }^{6}$ Department of Physiology, Fourth Military Medical University, Xi' an, China, ${ }^{7}$ Center \\ of Teaching Experiment, School of Basic Medical Science, Fourth Military Medical University, \\ Xi'an, China, ${ }^{8}$ Department of Radiology, Second Affiliated Hospital, Fourth Military Medical \\ University, Xi'an, China
}

Received February 2, 2015

Accepted June 12, 2015

On-line October 8, 2015

\section{Summary}

Proximal resistance vessels, such as the mesenteric arteries, contribute substantially to the peripheral resistance. The reactivity of resistance vessels to vasoactive substance like natriuretic peptides plays an important role in the regulation of blood pressure. In current study, we investigated the reactivity of mesenteric arteries to atrial natriuretic peptide (ANP), a well known vasodilating factor, in spontaneously hypertensive rats (SHR), as well as the effects of exercise training on it. As a result, ANP-induced vasorelaxation was attenuated in SHR with significantly increased phosphodiesterase type 5 (PDE5), and decreased cGMP/ANP ratio, compared with WKY rats as control. Intriguingly, the decreased reactivity to ANP in SHR was markedly reversed by exercise training. In addition, ANP resistance of in vitro mesenteric arteries was diminished by sildenafil a potent selective inhibitor of PDE5. In conclusion, ANP resistance occurs in resistance vessels of SHR, suggesting predisposition to hypertension, which can be reversed by exercise.

\section{Key words}

Natriuretic peptide • Hypertension • Resistance vessels • cGMP

\section{Corresponding authors}

B.-Y. Chen, Department of Radiology, Second Affiliated Hospital, Fourth Military Medical University, Xi'an, China. E-mail: pclamper@163.com

and

H.-F. Zhang, Center of Teaching Experiment, School of Basic Medical Science, Fourth Military Medical University, Xi'an, China. E-mail: hfzhang@fmmu.edu.cn and

M.-Z. Zhu, Department of Physiology, Fourth Military Medical University, Xi'an, China. E-mail: mz_zhu@fmmu.edu.cn

\section{Introduction}

Hypertension is an important public health issue worldwide because of its high prevalence and concomitant increase in disease risk. It has been estimated that $29 \%$ of the world's adult population, or approximately 1.56 billion people, will have hypertension by 2025 (Lemogoum 2014, Shrivastava et al. 2014). Childhood hypertension is a serious public health problem worldwide, especially in China. Epidemiological studies have suggested that childhood hypertension could track into adulthood. In addition, hypertension in 
childhood was strongly associated with increased rates of morbidity and mortality during adulthood (Xi et al. 2014). Therefore, it is important to clarify the underlying mechanism that involved with the "starting-points" for hypertension.

Hypertension is a disorder characterized by persistent increase of vascular tone especially in the resistance vasculature. Both functional (i.e., increased vascular smooth muscle sensitivity) and structural changes (i.e., the ratio of medial thickness to lumen diameter) in the resistance arteries have been found in spontaneously hypertensive rat (SHR), as well as in human primary hypertension (Mulvany 1991). These abnormalities are usually closely correlated with blood pressure, but it is a question whether the abnormalities cause or are the consequences of the increased blood pressure. Importantly, these alterations in proximal resistance arteries occur so early in life that they might serve as major "starting-points" for hypertension. Thus, it suggested that the abnormalities of vascular smooth muscle activity, and of hemodynamics in the resistance arteries, particularly in early phases of primary hypertension, play an important role in genesis and development of hypertension (Folkow 1997).

Atrial natriuretic peptide (ANP), a cardiovascular hormone, mainly secreted by heart atria in response to stretching forces induces diuretic, natriuretic and vasorelaxant effects and plays a major role in the homeostasis of blood pressure as well as of water and salt balance (De Vito 2014). In vitro studies provide evidences that ANP is a potent vasodilating agent on rat abdominal aorta, pulmonary artery, and mesenteric resistance arteries via cGMP-PKG pathway (Feng et al. 1999, Iwatani et al. 2007).

It was reported that in such pathophysiological process as microgravity (Behnke et al. 2008), and endothelium injuries (Iwatani et al. 2007), the vasoconstriction of mesenteric resistance arteries is altered, and these changes are associated with the levels of blood pressure. However, the responsivity of resistance arteries in early phases of primary hypertension, as well as its functional role in hypertension is largely unknown. Thus, the current study is delivered to test the sensitivity changes of mesenteric arteries to natriuretic peptide in spontaneous hypertensive rat in order to reveal a novel mechanism of hypertension. Furthermore, intervention effects of exercise were evaluated in the view of transformation medicine to provide experimental evidences for prevention and therapeutics by the means of exercise training.

\section{Materials and Methods}

\section{Reagents}

All chemicals and reagents were purchased from Sigma (Sigma, St. Louis, MO, USA) unless indicated otherwise.

\section{Animals}

SHR (Spontaneous Hypertensive Rats) and WKY (Wistar Kyoto) rats were housed in separate cages in a temperature-controlled room $\left(22-24{ }^{\circ} \mathrm{C}\right)$ under a 12 -h light/12-h dark cycle with free access to food and water. All animal procedures described in this study were approved by the Committee on Animal Care of Xi'an Medical University.

\section{Animal grouping and exercise training protocols}

As shown in Figure 1, there were 60 animals assigned into 6 groups $(\mathrm{n}=10)$ : (1) 4-week-old WKY rats; (2) 4-week-old SHR; (3) 16-week-old WKY rats; (4) 16-week-old SHR; (5) 16-week-old WKY rats trained with exercise; (6) 16-week-old SHR trained with exercise.

WKY rats and SHR in the exercised groups were trained, free of loading, 5 days/week for 12 weeks in a 60-90 cm tank filled with approximately $50 \mathrm{~cm}$ depth of water at $33-35{ }^{\circ} \mathrm{C}$. Rats swam in groups of three or four animals. Rats swam for $15 \mathrm{~min}$ on the first day, and the swimming duration was then progressively increased to $60 \mathrm{~min} /$ day in 1 week.

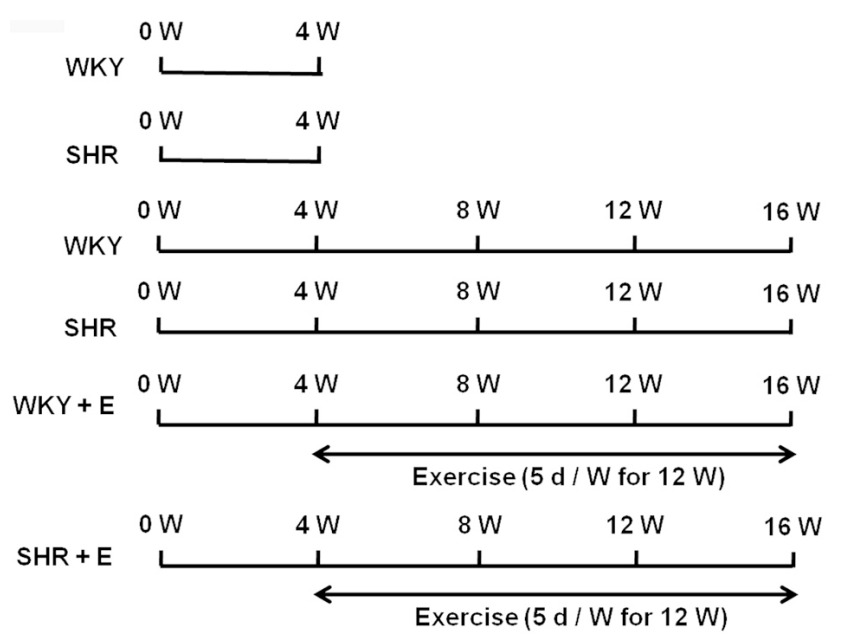

Fig. 1. Animal grouping and exercise training protocols. Sixty animals were assigned into 6 groups $(n=10)$. Spontaneously hypertensive rats (SHR), or WKY rats were trained for 12 weeks ( 5 days/week) by swimming or not trained as control. 


\section{Assessment of angiotasis}

Rats were anesthetized with sodium pentobarbital (30 mg/kg, i.p.). Mesenteric arteries were isolated from rats as described (Wang et al. 2011) and cut into rings. After obtaining a sustained and comparable contraction induced by NE (norepinephrine, $10^{-6} \mathrm{~mol} / \mathrm{l}$ ), the functional integrity of endothelium was assessed by recording the relaxation to $10^{-5} \mathrm{~mol} / \mathrm{l}$ acetylcholine. Vascular responses to ANP were determined by exposing mesenteric arterial segments to four concentrations of ANP $\left(10^{-9}, 10^{-8}, 10^{-7}, 10^{-6} \mathrm{~mol} / \mathrm{l}\right)$, and the resulting changes in isometric tension were recorded during $10 \mathrm{~min}$ after each concentration of ANP. For PED5 inhibition, sildenafil $(30 \mu \mathrm{mol} / \mathrm{l})$ was added into the bath solution 15 min before treatment of ANP. In analysis, the maximal contraction amplitude induced by norepinephrine $\left(10^{-6} \mathrm{~mol} / \mathrm{l}\right.$ was set as $100 \%$.

\section{Assessment of arterial blood pressure}

Rats were anaesthetized with sodium pentobarbital (30 mg/kg, i.p.). The arterial blood pressure was measured via a polyethylene catheter cannulated into the left carotid arteries. Arterial systolic and diastolic blood pressure (SBP and DBP) were continuously monitored via a data acquisition system (BL-420S system, Taimeng, Chengdu, China).

\section{Measurements of plasma cGMP and ANP}

Blood was collected in tubes containing EDTA, and was immediately placed on ice. Samples were then centrifuged at $2000 \mathrm{rpm}$ for $30 \mathrm{~min}$, plasma separated and stored at $-80^{\circ} \mathrm{C}$. Plasma ANP and cGMP were determined using commercially available kits (East Asia Immunotechnology Institute, General Hospital of People's Liberation Army of China, Beijing, China). The ratio of plasma cGMP to plasma ANP (cGMP/ANP) were calculated and used as in vivo markers of signaling sensitivity to ANP.

\section{Tissue PDE5 activity assays}

PDE activity was measured following previously reported methods (Gopal et al. 2001). Mesenteric arteries from rats were dissected and homogenized in $20 \mathrm{mmol} / 1$ Tris-HCl buffer at $\mathrm{pH} 7.2$, containing $0.2 \mathrm{mmol} / 1$ EGTA, $5 \mathrm{mmol} / \mathrm{l} \beta$-mercaptoethanol, $2 \%(\mathrm{v} / \mathrm{v})$ antiprotease cocktail, $1 \mathrm{mmol} / 1 \mathrm{PMSF}, 0.1 \%$ Triton X-100, $5 \mathrm{mmol} / 1$ $\mathrm{MgCl}_{2}$ using a glass homogeniser on ice. The homogenates were centrifuged at $14000 \times \mathrm{g}$ for $30 \mathrm{~min}$ at $4{ }^{\circ} \mathrm{C}$, the pellets resuspended in the homogenization buffer and centrifuged at $14000 \times \mathrm{g}$ for $30 \mathrm{~min}$ at $4{ }^{\circ} \mathrm{C}$. The first and the second supernatants were pooled and used for further analysis.

Reactions were initiated by addition of tissue homogenate to a reaction mixture containing $40 \mathrm{mmol} / \mathrm{l}$ Mops (pH 7), 0.8 mmol/l EGTA, 15 mmol/1 magnesium acetate, $2 \mathrm{mg} / \mathrm{ml}$ bovine serum albumin, $\left[{ }^{3} \mathrm{H}\right]$ cGMP (80000-150000 cpm per assay tube) and varying concentrations of unlabeled cGMP, respectively. Reactions mixtures were incubated at $30^{\circ} \mathrm{C}$ for $15 \mathrm{~min}$. A mixture with the following ingredients was added to terminate the reaction: $50 \mathrm{mmol} / \mathrm{l}$ EDTA, $30 \mathrm{mmol} / \mathrm{l}$ theophylline, $10 \mathrm{mmol} / \mathrm{l}$ cGMP in $100 \mathrm{mmol} / \mathrm{l}$ Tris (pH 7.5). Snake venom 50-nucleotidase (Sigma) (200 mg) was added to the assay mixture and incubated for $10 \mathrm{~min}$ at $30{ }^{\circ} \mathrm{C}$. Assay samples were diluted in $1 \mathrm{ml}$ of dilution solution $(0.15 \mathrm{mmol} / \mathrm{l}$ EDTA containing $100 \mathrm{mmol} / \mathrm{l}$ guanosine) and applied to QAE-Sephadex columns $(8 \times 33 \mathrm{~mm})$ preequilibrated in $20 \mathrm{mmol} / 1$ ammonium formate buffer $(\mathrm{pH}$ 7.4). The eluates were collected and the amount of $\left[{ }^{3} \mathrm{H}\right]$ guanosine was determined using commercially available kits (East Asia Immunotechnology Institute, General Hospital of People's Liberation Army of China, Beijing, China).

\section{Western blotting}

For preparation of tissue lysates, mesenteric arteries from rats were dissected and washed with icecold PBS and homogenized with RIPA buffer with $150 \mathrm{mmol} / 1 \mathrm{NaCl}$ as described (Yu et al. 2010). Cell lysates were cleared at $20000 \times \mathrm{g}$ for $10 \mathrm{~min}$. After adjustment of protein concentration, the lysates were boiled in SDS sample loading buffer for $5 \mathrm{~min}$ and separated by SDS-polyacrylamide gel electrophoresis (PAGE, 4-15\%, BioRad). Gels were blotted on a polyvinylidene difluoride (PVDF) membrane (Immobilon P; Millipore, Bedford, MA, USA) and stained with the indicated first antibody anti-rat PDE5 (1:1000 dilution, Cell Signaling Technology, Inc., Danvers, MA, USA), and anti-human $\beta$-actin (40 ng/ml, Santa Cruz Inc., Santa Cruz, CA, USA). Antibody binding was detected with horseradish peroxidase (HRP)coupled secondary antibody followed by chemiluminescence detection (ECL Plus; Amersham Pharmacia, Uppsala, Sweden).

\section{Statistical analysis}

Quantitative data are presented as individual data plots or as mean plus standard deviation. Statistical 
analyses were done with SPSS, version 15.0 (SPSS Science, Chicago, IL, USA). Statistical significances of overall differences between multiple groups were analyzed by one-way ANOVA analysis. Differences between two independent groups were analyzed by the student $t$ test. $P$ values less than 0.05 were considered significant.

\section{Results}

Exercise training reversed elevation of blood pressure in spontaneously hypertensive rats

The body weights of the young (4-week-old) rats were not significantly different $(P>0.05$; Student's unpaired $t$ test) between the two strains (SHR $80.1 \pm 10.3 \mathrm{~g}$; WKY 75.6 $\pm 9.2 \mathrm{~g} ;$ mean $\pm \mathrm{SD}, \mathrm{n}=10$ ). In the adult (16-week-old) groups, the mean weights were again not significantly different $(P>0.05)$ between the two strains (SHR 324.6 $\pm 42.7 \mathrm{~g}$; WKY 358.7 $\pm 45.2 \mathrm{~g}, \mathrm{n}=10$ ).

As shown in Figure 2A, the mean carotid arterial pressures of 4-week-old animals were not significantly different between the WKY rats and SHR. However, in the adult (16-week-old) animals, SHR demonstrated significantly higher mean carotid arterial pressures compare with even-aged WKY rats $(P<0.01)$. As shown in Figure 2B, exercise training significantly decreased the elevation mean carotid arterial pressures in SHR $(P<0.05)$.
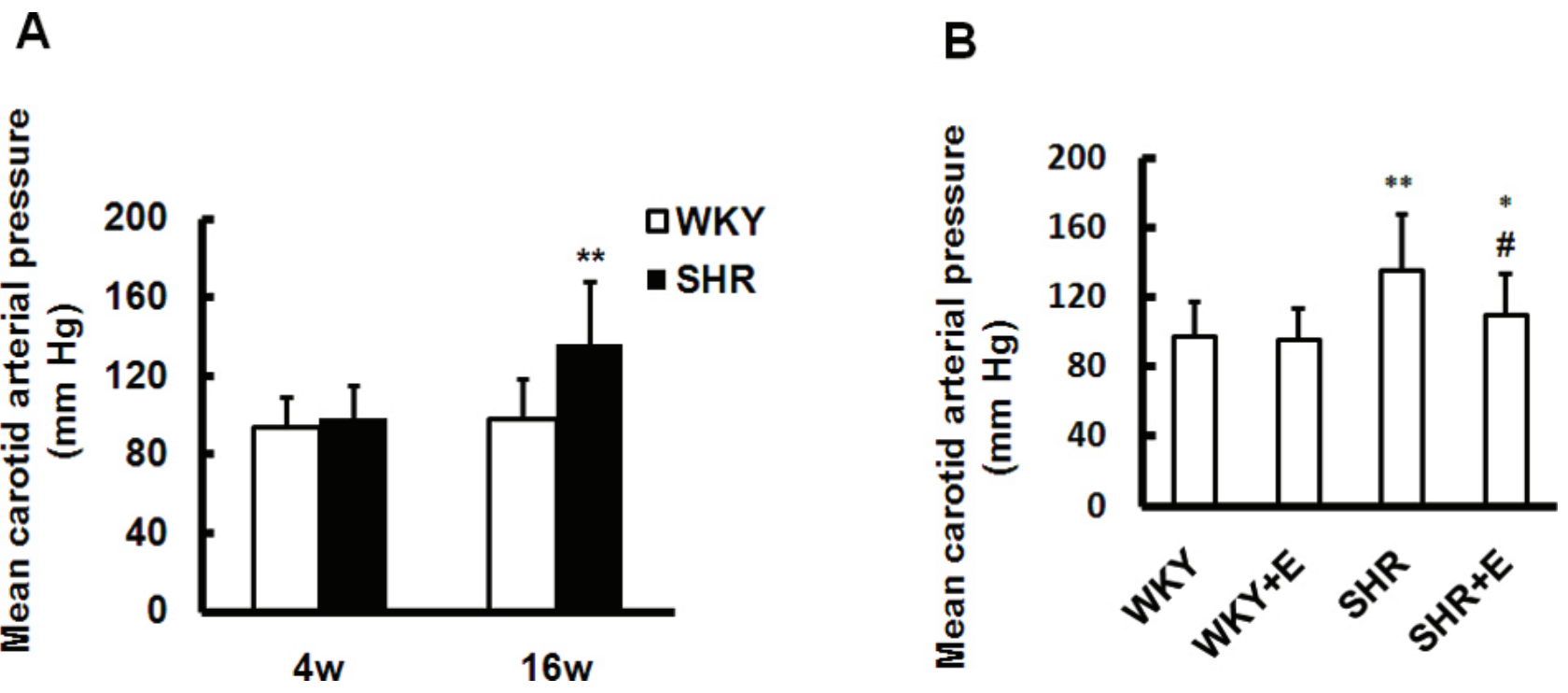

Fig. 2. Exercise training reversed elevation of arterial blood pressure in spontaneously hypertensive rats (SHR). Systolic and diastolic blood pressure was measured by arterial cannulization in the left carotid artery of WKY rats or SHR. Mean carotid arterial pressures were calculated automatically. (A) Mean carotid arterial pressures in age-matched WKY rats or SHR. (B) Mean carotid arterial pressures in 16-week-old WKY rats and SHR with or without exercise training. $* P<0.05, * * P<0.01$ versus WKY, $\# P<0.05$ versus SHR, $\mathrm{n}=10$

Sensitivity of resistant vessels to ANP was decreased in SHR

Although it has been extensively scrutinized, the factors involved in the initiation and development of hypertension in SHRs remains unresolved (Komolova et al. 2012). Just as previously reported, the blood pressure was not yet elevated in 4-week-old SHR (Komolova et al. 2012, Török et al. 2006, Corbett et al. 2007). In order to know whether changes of vascular response to ANP is the "initiator" or "result" of hypertension, we tested the vasorelaxing properties induced by ANP in both juvenile (before hypertension) and adult (hypertension) SHR.

In juvenile (4-week-old) WKY or spontaneously hypertensive rats, ANP induced a dose dependent relaxation of mesenteric arteries precontracting by $10^{-6}$ mol/1 NE. Mesenteric arteries from 4-week-old SHR demonstrated attenuated response to ANP at the dose of $10^{-8} \mathrm{~mol} / 1$ or $10^{-9} \mathrm{~mol} / 1$ compared with age-matched WKY rats (Fig. 3A). Differently, relaxation of mesenteric arteries from 16-week-old SHR was even attenuated to ANP at the dose from $10^{-9} \mathrm{~mol} / 1$ to $10^{-6} \mathrm{~mol} / \mathrm{l}$ (Fig. 3B). Accordingly, plasma cGMP/plasma ANP ratio was markedly reduced in juvenile (4-week-old) SHR (Fig. 3C) and even decreased in the adult (16-week-old) group (Fig. 3D), suggesting impaired cGMP generation despite high ANP stimulation. The fact that ANP resistance occurred before the formation of hypertension indicated predisposition to hypertension. 

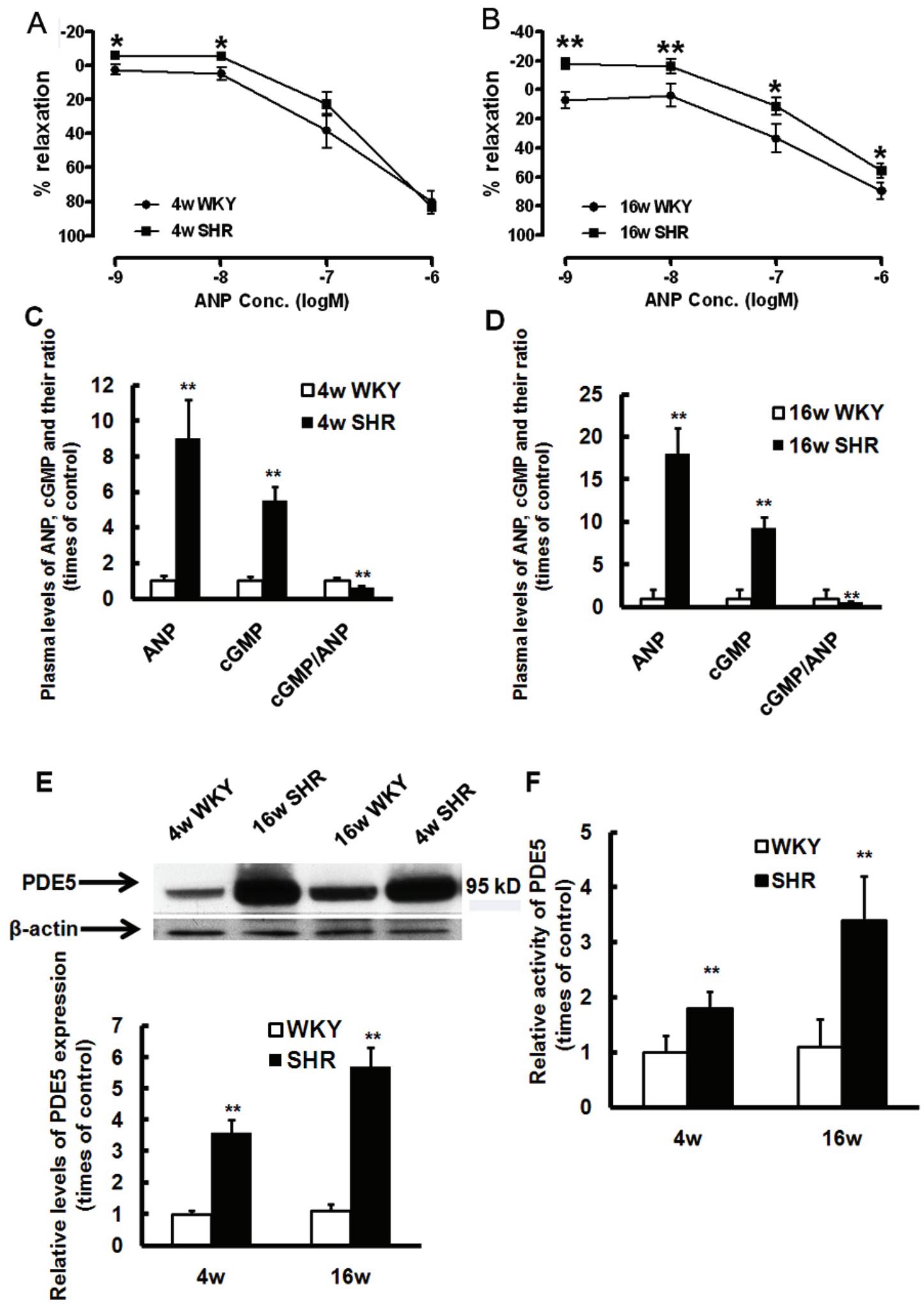

Fig. 3. ANP resistance of mesenteric arteries in spontaneously hypertensive rats (SHR). (A) Relaxation curves of mesenteric arteries from juvenile (4-week-old) WKY or spontaneously hypertensive rats. $n=10$. (B) Relaxation curves of mesenteric arteries from adult (16-week-old) WKY or spontaneously hypertensive rats. $n=10$. (C) Plasma levels of ANP, cGMP and the ratio of cGMP/ANP from juvenile (4-week-old) WKY, and spontaneously hypertensive rats. Data were from 5 independent experiments. The levels of ANP, cGMP and the ratio of CGMP/ANP from WKY rats as control were arbitrarily set as 1. (D) Plasma levels of ANP, cGMP and the ratio of cGMP/ANP from adult (16-week-old) WKY, and spontaneously hypertensive rats. Data were from 5 independent experiments. The levels of ANP, CGMP and the ratio of CGMP/ANP from WKY rats as control were arbitrarily set as 1 . (E) The expression levels of PDE5 in mesenteric arteries from rats were determined by western blotting. Data were from 3 independent experiments. (F) The activity of PDE5 in mesenteric arteries from rats. Data were from 3 independent experiments. The expression levels or activity of PDE5 in WKY rats were arbitrarily set as $1 . * P<0.05, * * P<0.01$ versus $\mathrm{WKY}$ 
A

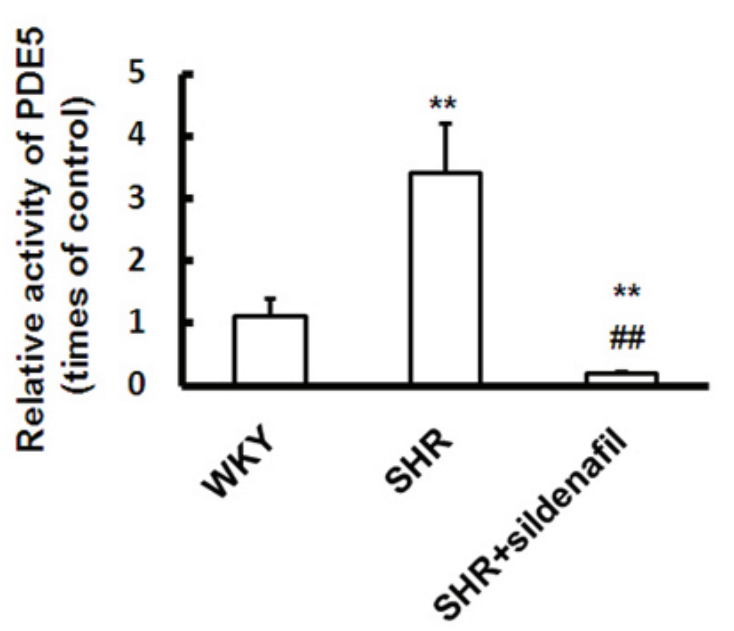

C

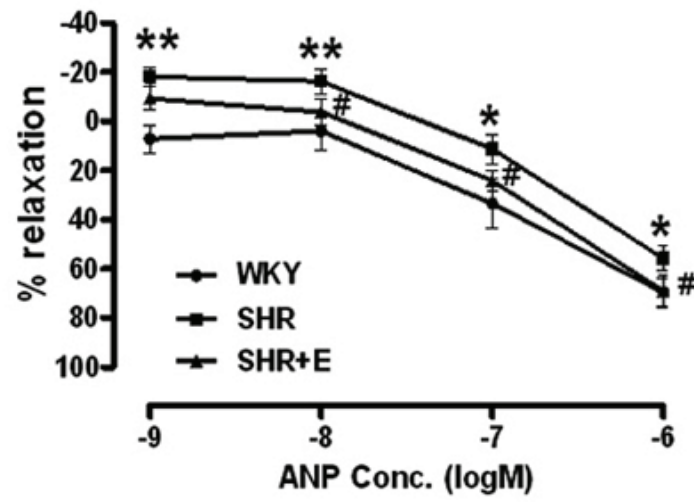

E

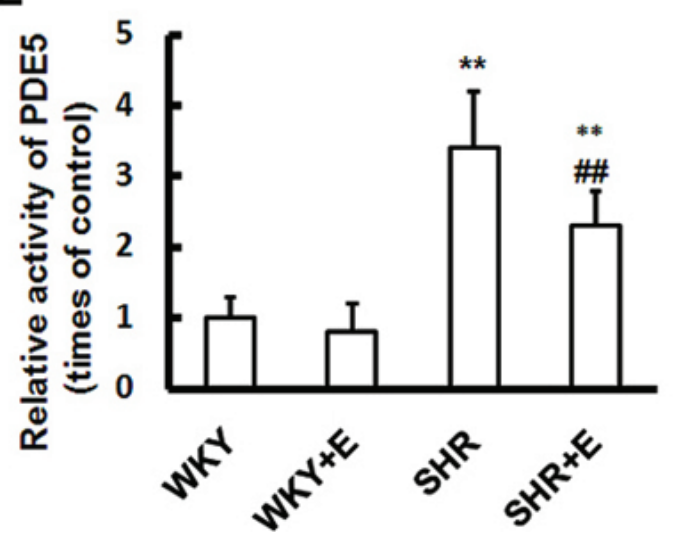

B
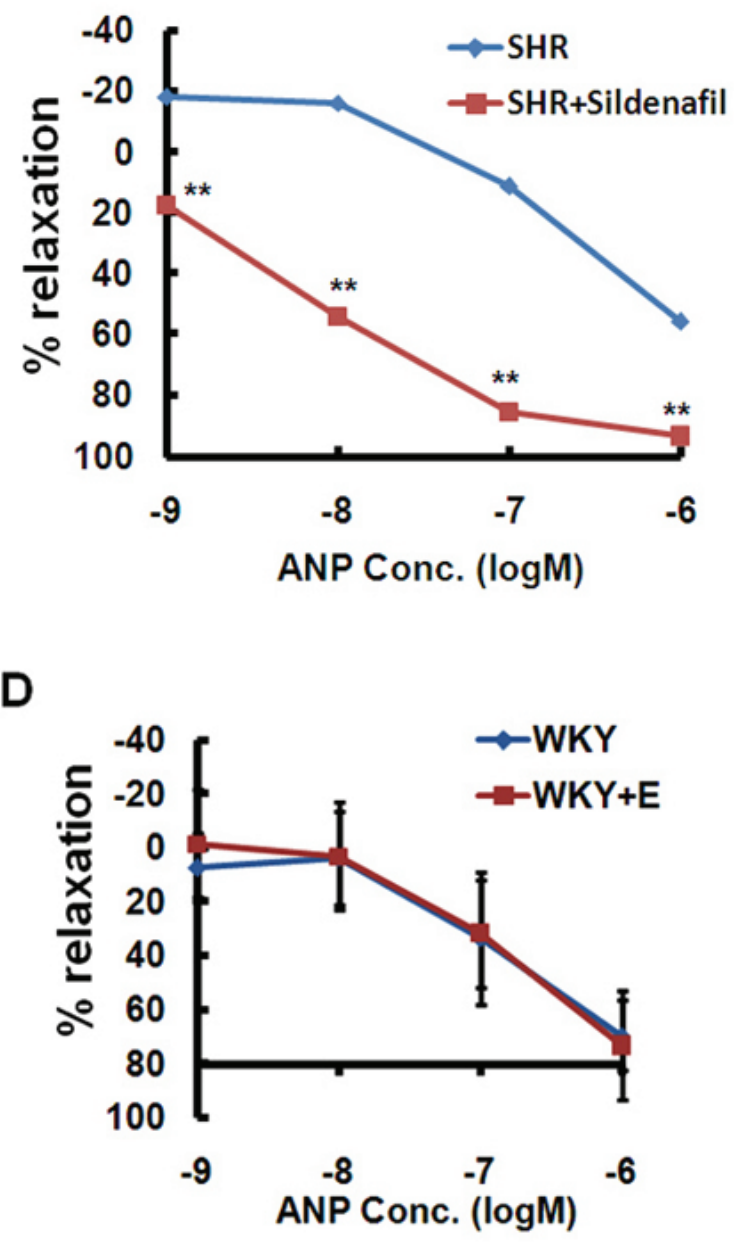

$\mathbf{F}$

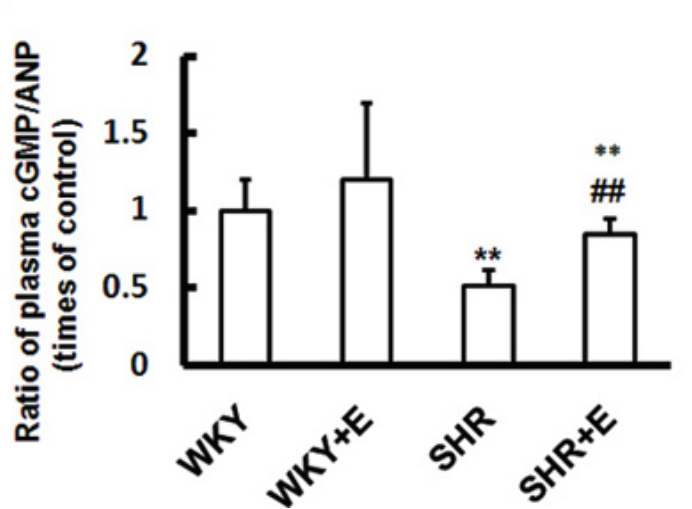

Fig. 4. Exercise or sildenafil reversed ANP resistance of mesenteric arteries in spontaneously hypertensive rats (SHR). (A) Mesenteric arteries from rats were dissected and homogenized, and the activity of PDE5 was determined. Data were from 3 independent experiments. The activity of PDE5 in WKY rats were arbitrarily set as 1. (B) Relaxation curves of mesenteric arteries from adult (16-week-old) spontaneously hypertensive rats. $n=10$. (C) Relaxation curves of mesenteric arteries from WKY, SHR, or SHR with exercise training (SHR+E). $n=10$. (D) Relaxation curves of mesenteric arteries from WKY rats with or without exercise training. $n=10$. (E) Mesenteric arteries from WKY rats and SHR with or without exercise training were dissected and homogenized, and the activity of PDE5 was determined respectively. Data were from 3 independent experiments. The activity of PDE5 in WKY rats without exercise training was arbitrarily set as 1 . (F) The plasma levels of cGMP and ANP were detected, and the ratios of plasma cGMP/ANP was calculated. Data were from 3 independent experiments. The ratios of plasma cGMP/ANP in WKY rats without exercise training were arbitrarily set as $1 . * P<0.05, * * P<0.01$ versus WKY, \# $P<0.05, \# \#<<0.01$ versus SHR 
Increased activity of PDE5 was culpable to ANP resistance in $S H R$

NPRA-cGMP (natriuretic peptide receptor A-cyclic guanosine monophosphate) pathway is the classical signal that involved with vasorelaxing effects of ANP, and cGMP acts as the second messenger. ANP binds to the NPR-A coupled to particulate guanylate cyclase, eliciting their biologic action by increasing cGMP synthesis, and cGMP is degraded by phosphodiesterases such as PDE5 (Kuhn 2003). For the sake of reveal the underlying mechanism of ANP resistance in hypertension, we tested the expression as well as activity of PDE5 in mesenteric arteries.

As shown in Figure 3E, the expression of PDE5 was significantly enhanced in young (4-week-old) SHR compared with WKY as control, and it was even increased in the adult (16-week-old) SHR. Accordingly, the activity of PDE5 was also significantly increased in both young and adult SHR (Fig. 3F). Importantly, sildenafil, an inhibitor of PDE5, efficiently suppressed the activity of PDE5 (Fig. 4A), and attenuated the ANP resistance in SHR (Fig. 4B).

\section{ANP resistance was alleviated by physical exercise}

Experimental studies and clinical observations have clearly showed that physical activity lowers blood pressure in both normal and hypertensive subjects by improving insulin sensitivity, facilitating arterial vasorelaxation to insulin ( $\mathrm{Li}$ et al. 2009). We thus speculate that exercise might also affect the ANP sensitivity of resistance artery.

Just as supposed, exercise significantly potentiated ANP's vasorelaxing effects, facilitated arterial vasorelaxation to ANP in SHR (Fig. 4C) but not in WKY rats as control (Fig. 4D). Importantly, exercise suppressed the enhanced activity of PDE5 in SHR (Fig. 4E), and increased in ratio of cGMP/ANP (Fig. 4F). This finding suggested a novel mechanism involved in the antihypertensive effects of exercise: improving the vascular sensitivity to ANP.

\section{Discussion}

In the current study we found that ANP resistance induced by the dysfunction of NPR-cGMP pathway in hypertension. This conclusion is based on the following findings: (1) ANP-induced relaxation of mesenteric artery was significantly attenuated in 4-weekold SHR, and the relaxation was even deteriorated in 16-week-old SHR (Fig. 3A, B). (2) The ratio of plasma cGMP/ANP was significantly decreased in SHR (Fig. 3C, D). (3) The expression as well as activity of PDE5, an important regulator of NPR-cGMP pathway, was significantly increased in SHR (Fig. 3E, F). (4) Exercise (Fig. 4C) or sildenafil (Fig. 4B), an inhibitor of PDE5, was able to reverse ANP resistance in SHR by suppressing PDE5.

Although the elevation of ANP as well as exaggerated production of cGMP in SHR has ever been reported early (Tremblay et al. 1993, Martel et al. 2010), natriuretic peptide resistance was first raised as a novel concept by Forfia et al. (2007) in their report on the brain natriuretic peptide resistance in heart failure. In current study, we found ANP resistance in hypertension. Importantly, the ANP resistance of mesenteric arteries occurs on the early stage of hypertension (4-week-old SHR). It suggests that reduced sensitivity of resistance blood vessels to ANP might culpable for the predisposition to hypertension. Allowing for the vasorelaxing, diuretic and natriuretic actions of ANP (De Vito 2014), the even decreased ANP sensitivity in 16-week-old SHR raises the possibility that ANP resistance plays an important role in the development of hypertension. It is well known that insulin resistance has comprehensive effects in metabolic syndrome and cardiovascular diseases and involved damage of kinds of organs such as heart (Zhang 2014), blood vessels (Paneni et al. 2013, Cubbon et al. 2013). Similarly, ANP resistance might not be limited within the mesenteric arteries, but occurs in systematically involving heart, artery, kidney liver or brain. This is worthy of further investigation.

NPRA-cGMP signal is a classical pathway induced by ANP. As shown in Figure 3 and Figure 4, PDE5 plays a vital role in ANP resistance. PDE5, a subtype phosphodiesterase decomposing cGMP, acts a "switch" of NPRA-cGMP signal. Abnormally enhanced PDE5 induces "off" status of NPRA-cGMP signal resulting in ANP resistance. In current study we found that sildenafil, a potent selective inhibitor of PDE5 reverses ANP resistance in hypertension (Fig. 4B). This result produces evidence for the functional role of PDE5 in ANP resistance. Klaiber et al. (2011) previously reported a cGMP independent signaling pathway. Whether ANP resistance is also related to the cGMP independent pathway is unknown and needs investigations. 


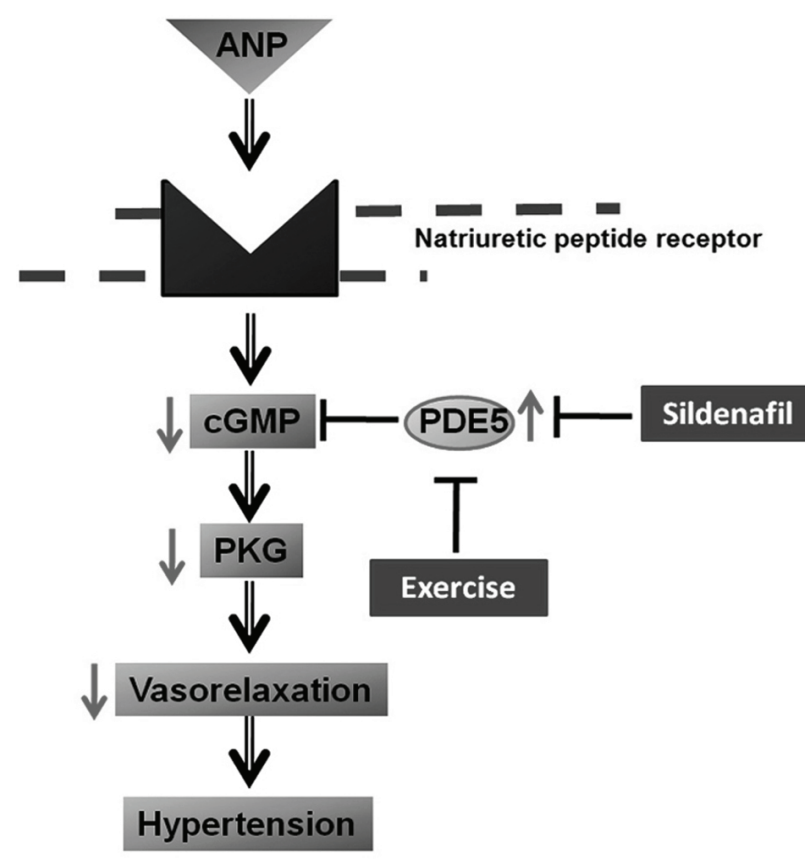

Fig. 5. A schematic representation of proposed signaling pathways whereby enhancement of PDE5 induced ANP resistance, which was reversed by exercise or sildenafil. cGMP, cyclic guanosine 3',5'-monophosphate; PKG, cGMP-dependent protein kinase G; PDE5, phosphodiesterase type 5.

Physical exercise elicits salutary actions on chronic metabolic diseases (Lancaster and Febbraio 2014). Li et al. (2009) reported that aging-associated insulin resistance was reversed by exercise in rats. We demonstrate evidence in current study that exercise alleviates ANP resistance of mesenteric arteries in SHR. This finding gives clues to a novel mechanism of exercise-associated effects against hypertension. Importantly, we found that effects of exercise were associated with suppression of PDE5, which acts as a switch of NPRA-cGMP pathway.

Taken together, evidence from the current study supports a key role of PDE5 in modulating the vascular sensitivity to ANP by deactivating cGMP. Abnormally enhanced PDE5 is culpable for the desensitization of NPR-cGMP-PKG signaling pathway resulting in ANP resistance, which can be reversed by exercise or sildenafil (Fig. 5).

\section{Conflict of Interest}

There is no conflict of interest.

\section{Acknowledgements}

This study was supported by the National Natural Science Foundation of China (No. 81270329, 81470438, 81300190, 81270330, 31271220). Natural Science Funds of Shaan'xi Province (No. 2014JM4166, 2014K11-0101-07, 14JK1616, 2013KJXX-89).

\section{References}

BEHNKE BJ, ZAWIEJA DC, GASHEV AA, RAY CA, DELP MD: Diminished mesenteric vaso- and venoconstriction and elevated plasma ANP and BNP with simulated microgravity. J Appl Physiol 104: 1273-1280, 2008.

CORBETT EK, MARY DA, MCWILLIAM PN, BATTEN TF: Age-related loss of cardiac vagal preganglionic neurones in spontaneously hypertensive rats. Exp Physiol 92: 1005-1013, 2007.

CUBBON RM, MERCER BN, SENGUPTA A, KEARNEY MT: Importance of insulin resistance to vascular repair and regeneration. Free Radic Biol Med 60: 246-263, 2013.

DE VITO P: Atrial natriuretic peptide: an old hormone or a new cytokine? Peptides 58: 108-116, 2014.

FENG HS, ZANG YM, ZHU MZ, PEI JM, WANG YM, WANG L, SHI PT: Comparison of vasorelaxing actions of vasonatrin peptide, C-type natriuretic peptide and atrial natriuretic peptide. (in Chinese) Sheng Li Xue Bao 51: 515-520, 1999.

FOLKOW B: Comments on "endpoints in hypertension": peripheral resistance vessels - though mainly on their involvement as "starting-points". Blood Press Suppl 2: 34-38, 1997.

FORFIA PR, LEE M, TUNIN RS, MAHMUD M, CHAMPION HC, KASS DA: Acute phosphodiesterase 5 inhibition mimics hemodynamic effects of B-type natriuretic peptide and potentiates B-type natriuretic peptide effects in failing but not normal canine heart. J Am Coll Cardiol 49: 1079-1088, 2007.

GOPAL VK, FRANCIS SH, CORBIN JD: Allosteric sites of phosphodiesterase-5 (PDE5). A potential role in negative feedback regulation of cGMP signaling in corpus cavernosum. Eur J Biochem 268: 3304-3312, 2001.

IWATANI Y, NUMA H, ATAGI S, TAKAYAMA F, MIO M, KAWASAKI H: Mechanisms underlying enhanced vasodilator responses to various vasodilator agents following endothelium removal in rat mesenteric resistance arteries. (in Japanese) Yakugaku Zasshi 127: 729-733, 2007. 
KLAIBER M, DANKWORTH B, KRUSE M, HARTMANN M, NIKOLAEV VO, YANG RB, VÖLKER K, GASSNER B, OBERWINKLER H, FEIL R, FREICHEL M, GROSCHNER K, SKRYABIN BV, FRANTZ S, BIRNBAUMER L, PONGS O, KUHN M: A cardiac pathway of cyclic GMP-independent signaling of guanylyl cyclase A, the receptor for atrial natriuretic peptide. Proc Natl Acad Sci U S A 108: 18500-18505, 2011.

KOMOLOVA M, FRIBERG P, ADAMS MA: Altered vascular resistance properties and acute pressure-natriuresis mechanism in neonatal and weaning spontaneously hypertensive rats. Hypertension 59: 979-984, 2012.

KUHN M: Structure, regulation, and function of mammalian membrane guanylyl cyclase receptors, with a focus on guanylyl cyclase-A. Circ Res 93: 700-709, 2003.

LANCASTER GI, FEBBRAIO MA: The immunomodulating role of exercise in metabolic disease. Trends Immunol 35: 262-269, 2014.

LEMOGOUM D: Challenge for hypertension prevention and control worldwide: he time for action. J Clin Hypertens (Greenwich) 16: 554-556, 2014.

LI QX, XIONG ZY, HU BP, TIAN ZJ, ZHANG HF, GOU WY, WANG HC, GAO F, ZHANG QJ: Aging-associated insulin resistance predisposes to hypertension and its reversal by exercise: the role of vascular vasorelaxation to insulin. Basic Res Cardiol 104: 269-284, 2009.

MARTEL G, HAMET P, TREMBLAY J: Central role of guanylyl cyclase in natriuretic peptide signaling in hypertension and metabolic syndrome. Mol Cell Biochem 334: 53-65, 2010.

MULVANY MJ: Are vascular abnormalities a primary cause or secondary consequence of hypertension? Hypertension 18 (Suppl I): I52-I57, 1991.

PANENI F, BECKMAN JA, CREAGER MA, COSENTINO F: Diabetes and vascular disease: pathophysiology, clinical consequences, and medical therapy: part I. Eur Heart J 34: 2436-2443, 2013.

SHRIVASTAVA SR, SHRIVASTAVA PS, RAMASAMY J: The determinants and scope of public health interventions to tackle the global problem of hypertension. Int J Prev Med 5: 807-812, 2014.

TÖRÖK J, KOPRDOVÁ R, CEBOVÁ M, KUNES J, KRISTEK F: Functional and structural pattern of arterial responses in hereditary hypertriglyceridemic and spontaneously hypertensive rats in early stage of experimental hypertension. Physiol Res 55 (Suppl 1): S65-S71, 2006.

TREMBLAY J, HUOT C, WILLENBROCK RC, BAYARD F, GOSSARD F, FUJIO N, KOCH C, KUCHEL O, DEBINSKI W, HAMET P: Increased cyclic guanosine monophosphate production and overexpression of atrial natriuretic peptide A-receptor mRNA in spontaneously hypertensive rats. J Clin Invest 92: 2499-2508, 1993.

WANG Y, CHENG KK, LAM KS, WU D, WANG Y, HUANG Y, VANHOUTTE PM, SWEENEY G, LI Y, XU A: APPL1 counteracts obesity-induced vascular insulin resistance and endothelial dysfunction by modulating the endothelial production of nitric oxide and endothelin-1 in mice. Diabetes 60: 3044-3054, 2011.

XI B, LI H, LI S, MI J: Recent prevalence of hypertension among Chinese children and adolescents based on 2010 China national blood pressure references. Int J Cardiol 174: 870-871, 2014.

YU J, BULK E, JI P, HASCHER A, TANG M, METZGER R, MARRA A, SERVE H, BERDEL WE, WIEWROTH R, KOSCHMIEDER S, MÜLLER-TIDOW C: The EPHB6 receptor tyrosine kinase is a metastasis suppressor that is frequently silenced by promoter DNA hypermethylation in non-small cell lung cancer. Clin Cancer Res 16: 2275-2283, 2010.

ZHANG PY: Cardiovascular disease in diabetes. Eur Rev Med Pharmacol Sci 18: 2205-2214, 2014. 